\title{
Birth asphyxia among term neonates at korle-bu teaching hospital (kbth) in ACCRA
}

\author{
Abstract \\ Objectives: \\ A. To determine the incidence of birth asphyxia among term neonates in Korle-Bu \\ Teaching Hospital \\ B. To determine the outcome among asphyxiated term neonates in Korle-Bu Teaching \\ Hospital
}

Study Design: Hospital based retrospective study

Method: Data were extracted using records from the Neonatal Intensive Care Unit (NICU) from the 1st of April 2015 to 30th of September, 2015. Extracted data included; total number of admissions for term neonates, number of term asphyxiated neonates and outcomes, and birth weights. Results were analysed using the SPSS version 16.0 software.

Results: Total number of admissions to NICU over the 6 month period was 966, out of which $468(48.4 \%)$ were term neonates. Of the term neonates $283(61.8 \%)$ were admitted for asphyxia. The overall mortality for the period was $203(21.0 \%)$. The mean birth weight for term neonates with asphyxia was $3.00 \mathrm{~kg}(\mathrm{SD} \pm 0.52)$. Mortality occurred in $63(21.8 \%)$ of the term neonates with birth asphyxia.

Conclusion: Perinatal admission and mortality from birth asphyxia among term neonates is unfortunately high in Korle-Bu Teaching Hospital.

In practical terms if perinatal mortality is to be reduced in the developing world including Ghana, concerted efforts should be towards reducing birth asphyxia among term neonates. This can be done even with limited resources. Continuous upgrading of neonatal resuscitation skills and the identification of prenatal and intrapartum risk factors which are largely known, in order to target the 'Therapeutic Window' for the management of birth asphyxia could not be overemphasized.

Keywords: Birth asphyxia; neonate, mortality, term
Volume 7 Issue 6 - 2017

K Mumuni,Ali Samba, JD Seffah

Department of Obstetrics \& Gynaecology, University of Ghana School of Medicine and Dentistry, Ghana

Correspondence: Ali Samba, Department of Obstetrics \& Gynaecology, University of Ghana School of Medicine and Dentistry. P. O. Box 4326, Accra. Ghana, Tel 233267647367. Emailabmasa@yahoo.com

Received: November 26, 2016 | Published: August 14, 2017

\section{Introduction}

It is of significant importance to note that of the 130 million babies born every year, about 4 million die in the first 4 weeks of life, the neonatal period. ${ }^{1}$ A similar proportion of babies are stillborn, dying inutero during the last 3 months of pregnancy. ${ }^{2}$

Most neonatal deaths (99\%) arise in low-income and middle income countries and about half occur at home. The countries with the highest rates of neonatal mortality are mostly in Sub-Saharan Africa (14 of the 18 countries with NMRs $>45$ per 1000). ${ }^{1}$ Babies born in Sub-Saharan Africa have a very high risk of birth asphyxia and intrapartum stillbirth. ${ }^{3}$

The psychological and emotional stress that is brought to bear on the woman, the couple or the family when a perinatal mortality occurs is unimaginable. And similarly care-givers go through a lot of emotional stress when patients they have cared for prenatally lose their babies, though in the developing world this so often happens to the extent that care-givers soon get accustomed to perinatal mortality and that they become somewhat indifferent to it, unfortunately. The psychological and emotional trauma seems to be much less in cases of preterm neonatal deaths than in term neonatal deaths. Because part of the blame for extreme preterm losses is attributed to nature and correctly so, it must be added, since the distinction between late abortion and extreme preterm birth is very thin as evidenced by the fact that in West African countries, 28 weeks of gestation is set as the dividing line of viability and births before 28 weeks are termed miscarriage or abortion. ${ }^{4}$ Even when such babies are born with signs of life little is done for their survival because of lack of supportive equipment.

In the developing world, if neonatal deaths are to be reduced significantly, then it is among term babies who are already equipped to survive even with minimal resources. It is against this background that this study was conducted to look at birth asphyxia among term neonates since birth asphyxia is one of the common causes of perinatal mortality in the developing world. Undoubtedly the biggest challenge to reducing neonatal mortality is among preterm neonates simply because their extra uterine survival systems are not fully developed especially at extreme preterm. Their survival is largely dependent on postpartum support. To decipher modifiable intrapartum practices besides the administration of steroids and advance resuscitation by neonatologist, intrapartum care givers would have to take a look at asphyxia among term neonates. Themodifiable intrapartum factors could then be applied to all deliveries irrespective of maturity beyond the period of viability. This is to deal with intrapartum preventable birth asphyxia, Korle-Bu Teaching Hospital is a tertiary institution where supervised delivery takes place, with comprehensive obstetric 
care and NICU facilities. This will probably provide insight into the problem outside tertiary institutions.

\section{Method}

NICU admissions records of the KBTH from 1st of April 2015 to 30th of September, 2015, were retrieved and data extracted and analysed using the SPSS version 16.0 software. Extracted data included the following: Total number of admissions, the number of admissions with the diagnosis of birth asphyxia, gestational age at delivery of each baby, outcomes of asphyxiated term babies and birth weights of term asphyxiated babies. Simple frequencies and means were calculated and presented as tables.

\section{Results}

Total number of admissions to NICU over the 6 month period was 966 , out of which $468(48.4 \%)$ were term neonates. Of the term neonates $289(61.8 \%$ ) were admitted for asphyxia. The mean birth weight for term neonates with asphyxia was $3.00 \mathrm{~kg}(\mathrm{SD} \pm 0.52)$. The overall mortality over the period was $203(21.0 \%)$ and among term neonates with birth asphyxia was 63(21.8\%) (Tables $1 \& 2)$.

Table I Number of NICU admissions and proportions of term neonates + - asphyxia

\begin{tabular}{ll}
\hline Total number of NICU admissions & 966 \\
Number of admissions for Term Neonates & 468 \\
Number of admissions for Term Neonates with Birth Asphyxia & 289 \\
Percent Term Neonates with Birth Asphyxia & $61.8 \%$ \\
\hline Table 2 Outcome of term asphyxiated neonates at NICU & \\
\hline Total number of NICU admissions & 966 \\
Number of admissions for Term Neonates & 468 \\
Number of admissions for Term Neonates with Birth Asphyxia & 289 \\
Percent Term Neonates with Birth Asphyxia & $61.8 \%$ \\
\hline
\end{tabular}

One hundred and forty nine (149) of them were male and 140 female. There was no obvious congenital malformation recorded. The mean maternal age was 29.14(SD 0.54) with a mean parity of 1.99 (SD 0.94). The mean apgar score of the asphyxiated babies at 1 minute and 5 minutes were 3.81 (SD 1.42) and 4.43(SD 1.19) respectively. Of these asphyxiated babies $47.8 \%$ and $31.7 \%$ were admitted with a 1 and 5 minutes apgar scores of $\leq 3$ respectively. Overall $84 \%$ of asphyxiated babies had apgar scores of $\leq 5$ at 5 minutes.

The apgar scores at both 1 and 5 minutes had no significant association with birth weight, maternal age and parity, with $\mathrm{p}=$ values of 0.723 and $0.593 ; 0.141$ and $0.454 ; 0.104$ and 0.996 respectively (Table 3). Majority, 218/289(75.4\%) were delivered by caesarean, out of which $180(82.6 \%)$ were emergencies and 38/218(17.4\%) electives. $69 / 289(23.9 \%)$ were delivered spontaneously vaginally and $2 / 289(0.7 \%)$ delivered vaginally with assistance. The indications for emergency caesarean section consisted of failure to progress/ cephalopelvic disproption (36.1\%), fetal distress (28.3\%), severe pre-eclampsia with unfavourable cervix $(17.8 \%)$, abruptio placentae (10.6\%), and breech presentation (7.2\%).

For the elective caesarean sections the commonest indication were intrauterine fetal growth restriction and 2 previous caesarean sections both at $34.2 \%$ each, followed by previous myomectomy and hypertension with diabetes both at $15.8 \%$ each. Majority $258 / 298$ $(89.3 \%)$ of mothers had normal physical health, maternal conditions included hypertension disease; 12/289(4.2\%), chronic diabetes; 2/289(0.7\%), sickle cell disease; 3/289(1.0\%), Fibroids; $2 / 289(0.7 \%)$,
Gestational diabetes plus chronic hypertension; 4/289(1.4\%), chronic hypertension 3/29(1.0\%) and gestational diabetes; $1 / 289(0.3 \%)$.

There was no significant association between the 1 and 5 minute apgar scores and the type of delivery, indication for caesarean section and maternal disease (Table 3).

Table 3 Relationship between selected characteristics and APGAR scores

\begin{tabular}{|c|c|c|}
\hline Characteristics & APGAR Score & p-value \\
\hline \multirow{2}{*}{ Maternal age } & $I \min$ & $0 .|4|$ \\
\hline & 5 mins & 0.454 \\
\hline \multirow{2}{*}{ Parity } & $I \min$ & 0.104 \\
\hline & 5 mins & 0.996 \\
\hline \multirow{2}{*}{ Birth weight } & Imin & 0.723 \\
\hline & 5 mins & 0.593 \\
\hline \multirow{2}{*}{ Type of delivery } & Imin & 0.451 \\
\hline & 5 mins & $0.54 I$ \\
\hline \multirow{2}{*}{ Caesarean section indication } & Imin & 0.903 \\
\hline & 5 mins & 0.514 \\
\hline \multirow{2}{*}{ Maternal disease } & $I \min$ & 0.619 \\
\hline & 5 mins & 0.396 \\
\hline
\end{tabular}

\section{Discussion}

It is estimated that around $24 \%$ of all newborn deaths are caused by birth asphyxia resulting in a large proportion of stillbirths. ${ }^{4}$ According to the World Health Organization (WHO) between four (4) and nine (9) million newborns develop birth asphyxia each year. Of these, an estimated 1.2 million die and at least the same number develop severe consequences, such as epilepsy, cerebral palsy and developmental delay. ${ }^{5}$ The incidence of birth asphyxia varies widely because of the difficulty in exact diagnosis. Birth asphyxia has been traditionally said to exist when there is failure to initiate and sustain breathing at birth. In recent position statements by the American College of Obstetricians and Gynaecologist. ${ }^{6}$ and the American Academy of Pediatrics. $^{7}$ a neonate is labeled to be asphyxiated if the following conditions are satisfied:

a. Umbilical cord arterial $\mathrm{pH}$ less than 7;

b. Apgar Score of 0 to 3 for longer than 5 minutes;

c. Neonatal neurologic manifestations (e.g. seizures, coma, or hypotonic); and

d. Multisystem organ dysfunction e.g. cardiovascular, gastrointestinal, haematologic, pulmonary, or renal system

In most cases in the developing world the Apgar score is the main means of initial diagnosis before neonatal neurologic manifestations and multisystem organ dysfunction are diagnosed. Facilities for fetal blood $\mathrm{pH}$ and umbilical cord $\mathrm{pH}$ are generally lacking. However, it is well known that the Apgar score is a poor predictor of outcome and severity of perinatal asphyxia when used alone and is also not able to predict long-term neurological developmental disabilities. ${ }^{8}$ Indeed the Apgar Score was not intended to be a measure of perinatal asphyxia. ${ }^{9}$ And yet it remains largely the main tool in the developing world for the diagnosis of fetal asphyxia.

However, it has been shown that a low 5 minute Apgar score clearly confers an increased relative risk of cerebral palsy, reported to be as high as 20-to 100-fold over that of infants with a 5 minute Apgar score of 7 to $10 .{ }^{10-12}$ Population studies have also shown that when the score is less than 5 at 5 and 10 minutes confers an increased relative risk of cerebral palsy, and the degree of abnormality correlates with the risk of cerebral palsy. However it is reassuring that most infants 
with low Apgar scores will not develop cerebral palsy. ${ }^{13}$ It is noted that Apgar scores are affected by gestational age, maternal medications, resuscitation, and cardiorespiratory and neurologic conditions. ${ }^{13}$

Following improvements in primary and obstetric care in most industrialized countries, the incidence of birth asphyxia has reduced significantly and less than $0.1 \%$ newborn infants die of birth asphyxia. ${ }^{14}$ In the developing countries, rates of birth asphyxia are several folds higher, ranging from 4.6 per 1000 in Cape Town. ${ }^{15}$ to 26 per 1000 in Nigeria. ${ }^{16}$ and case fatality rates may be $40 \%$ or higher. ${ }^{17}$ In this study $61.8 \%$ of the total admissions to NICU was for birth asphyxia among term neonates and mortality occurred in $21.8 \%$ of these asphyxiated term neonates. The mean weight of these asphyxiated term neonates was $3.00 \mathrm{~kg}$ (SD 0.52).

These results are comparable to that in a multicentre prospective study involving 4267 deliveries in 8 countries over a 3 month period in East, Central and Southern Africa that found the overall incidence of asphyxia of the newborn was $22.9 \%$ while that associated with Low birth weight $(<2500 \mathrm{gm})$ was $29.3 \%$ compared with $21.5 \%$ among the normal birth weight babies. ${ }^{12}$ Antenatal and intrapartum risk factors for birth asphyxia have been identified to include, antepartum hospitalization, antenatal and intrapartum anaemia, antepartum haemorrhage, severe preeclampsia/ eclampsia, augmentation of labour with oxytocin, premature rupture of membranes, meconium staining of liquor amnii, vacuum extraction, caesarean section, low birth weight and malpresentation..$^{18}$ In other studies, the leading obstetrical risk factors for asphyxia were difficult delivery (particularly breech delivery), prolonged labour, cephalopelvic disproportion (CPD) and fetal distress. ${ }^{16}$ Asphyxia was also more common when a midwife or TBA delivered the child. ${ }^{16}$

In this study there was statistically significant relationship between the Apgar scores at 1 minute and 5 minutes and the type of delivery (vaginal, caesarean section, vacuum delivery), maternal disease and indication for caesarean section. This could be explained by the fact that only asphyxiated babies were studied. It is capital intensive to decrease perinatal mortality and morbidity by decreasing birth asphyxia in the preterm neonate. However, with limited resources and appropriate training in neonatal resuscitation perinatal morbidity and mortality among term neonates can be reduced significantly.

It has to be noted that early assessment of the degree of resulting hypoxic-ischaemic damage, can provide useful information for both clinical management and the potential use of cerebro-protective strategies. And the interval after resuscitation from hypoxic-ischaemia during which an intervention might be efficacious in reducing the severity of brain damage, is called the "therapeutic window". In full term infants, the therapeutic window is short and possibly not longer than 1 to 2 hours. ${ }^{8}$ It is probably much shorter in preterm infants.

In this study the time and kind of resuscitation was not analysed, however other studies found that although prompt resuscitation after delivery can prevent many of these deaths and disabilities, it is often not initiated or the procedures used are inadequate or wrong. ${ }^{19}$ It has also been shown that after neonatal resuscitation program courses, the number of patients with perinatal asphyxia and also the duration of hospitalization decreased significantly, and the first minute Apgar scores increased significantly. ${ }^{20}$

This study is limited by the short duration of data collected as records for the preceding 3 months before the study were not available and at the time of submission, only 6 months data was accessed. It did not also consider sociodemographic factors and intrapartum events such as type and timing of interventions, medications etc and also how exactly the diagnosis of asphyxia as well as its severity was made except for the use Apgar scores. All these could have implications on the results found especially the outcome. The authors recommend a prospective study to address these limitations.

\section{Conclusion}

Perinatal admission and mortality from birth asphyxia among term neonates is unfortunately high in Korle-Bu Teaching Hospital. Known risk factors from literature for perinatal asphyxia need to be highlighted in obstetric care units especially in the labour wards, so that the "Therapeutic Window" is not missed in such cases. Also regular neonatal resuscitation programs should be instituted for labour ward staff and neonatal care unit staff in both hospitals and maternity homes. By this approach perinatal asphyxia among term infants in particular may be reduced significantly.

\section{Acknowledgements}

None.

\section{Conflicts of interest}

None.

\section{References}

1. Lawn Joy E, Cousens Simon, ZupanJelka. 4 million neonatal deaths when, where, why? Lancet March. 2005;365(9462):891-900.

2. Zupan J, Aahman E. Perinatal mortality for the year 2000: estimates developed by WHO. World Health Organization, Geneva. 2005

3. Lawn JE, Shibuya K, Stein C. No cry at birth: global estimates of intrapartum stillbirths and intrapartum-related neonatal deaths. Bull World Health Organ. 2005;83(6):409-417.

4. WHO. World Health Report 2005: make every mother and child count. Geneva, Switzerland: World Health Organization. 2005.

5. Saving Newborn Lives. The state of the World's newborn: a report from saving newborn lives. Washington DC, USA. 2001;p.1-44.

6. Committee on Fetus and Newborn. American Academy of Paediatrics and Committee on Obstetric Practice. 1996;98:141-142.

7. Gilstrap LC, Oh W. EIK Groove Village (IL); American Academy of Paediatrics and American College of Obstetricians and Gynaecologists. 2002;pp.196-197.

8. Batool AzraHaider, Zulfiqar A, Bhutta. Birth Asphyxia in Developing countries: Current Status and Public Health implications. Curr Probl Pediatr Adolesc Health Care. 2006;36(5):178-188.

9. Papile LA. The Apgar Score in the 21st century. $N$ Engl $\mathrm{J} \mathrm{Med}$. 2001;344(7):519-520.

10. Ehrenstein V. Association of Apgar scores with death and neurologic disability. Clin Epidemiol. 2009;1:45-53.

11. Moster D, Lie RT, Irgens LM, et al. The association of Apgar score with subsequent death and cerebral palsy: a population-based study in term infants. J Pediatr. 2001;138(6):798-803.

12. Lie KK, Grøholt EK, Eskild A. Association of cerebral palsy with Apgar score in low and normal birth weight infants: population based cohort study. BMJ. 2001;341:c4990.

13. The Apgar Score. American Academy of Pediatrics committee on fetus and newborn and American College of Obstetricians and Gynecologist committee on obstetric practice. 2015. 
14. Badawi N, Kurinczuk JJ, Keogh JM, et al. Intrapartum risk factors for Newborn encephalopathy: the Western Australian case-control study. BMJ. 1998;317(7172):1554-1558.

15. Hall DR, Smith M, Smith J. Maternal factors contributing to asphyxia neonatarium. J Trop Pediatr. 1996;42(4):192-195.

16. Kinoti SN. Asphyxia of the newborn in East, Central and Southern Africa. East Afr Med J. 1993;70(7):422-433.

17. Bang AT, Bang RA. Diagnosis of causes of childhood deaths in developing countries by verbal autopsy: suggested criteria. The SEARCH Team. Bull World Organ. 1992;70(4):499-507.
18. Kaye D. Antenatal and Intrapartum risk factors for birth asphyxia among emergency obstetric referrals in Mulago. Hosp, Kampala, Uganda. E African Med J. 2003;80(3):140-143.

19. Essential Newborn Care-Report of a technical working group. WHO/ FRH/MSM/96.13. 1996;p.19.

20. Duran R, Aladaq N, Vatansever U, et al. The Impact of Neonatal Resuscitation Program courses on mortality and morbidity of Newborn infants with perinatal asphyxia. Brain Dev. 2008;30(1):43-46. 